\title{
Re-infection outcomes following one- and two-stage surgical revision of infected hip prosthesis in unselected patients: protocol for a systematic review and an individual participant data meta-analysis
}

Setor K Kunutsor ${ }^{1 *}$, Michael R Whitehouse ${ }^{1}$, Jason Webb², Andrew Toms ${ }^{3}$, lan Stockley ${ }^{4}$, Adrian Taylor ${ }^{5}$, Stephen Jones ${ }^{6}$, Matthew Wilson ${ }^{3}$, Ben Burston${ }^{7}$, Tim Board ${ }^{8}$, John-Paul Whittaker ${ }^{7}$, Ashley W Blom and Andrew D Beswick ${ }^{1}$

\begin{abstract}
Background: Several aggregate published reviews have compared the effectiveness of one- and two-stage surgical revision to prevent re-infection following prosthetic hip infection and have reported inconsistent results. In addition, there were several features of these previous reviews which limited the validity of the findings. In the absence of a well-designed clinical trial, we propose the Global Infection Orthopaedic Management (INFORM) collaboration, a worldwide collaborative systematic review and meta-analysis of individual participant data (IPD) to address the existing uncertainties.

Methods: Cohort studies (prospective or retrospective) and randomised controlled trials conducted in unselected patients with infection treated exclusively by one- or two-stage revision and reporting re-infection outcomes within 2 years of revision will be retrieved by searching the following databases: MEDLINE, EMBASE, Web of Science, Cochrane Database of Systematic Reviews, Cochrane Central Register of Controlled Trials and the WHO International Clinical Trials Registry Platform. Reference lists of relevant studies will be manually scanned and there will be email contact with investigators of grey literature and conference abstracts. Investigators will be invited to join the Global INFORM collaboration and share their individual level data. The primary outcome of the analyses will be incidence of re-infection within 2 years of commencement of revision surgery. Primary analyses will be conducted comparing the one-stage to the two-stage surgical revision. IPD analyses will be based on Cox proportional hazard (PH) models estimated for each study separately. Study-specific log hazard ratios will be combined using random-effects meta-analysis with fixed-effects meta-analysis in subsidiary analyses. Hazard ratios for re-infection according to different individual level characteristics such as sex, age groups, body mass index and comorbidities will also be assessed.
\end{abstract}

Discussion: The analyses will enable a consistent approach to the definition of re-infection outcomes, more detailed analyses under a broader range of circumstances and exploration of potential sources of heterogeneity and produce much more valid and precise estimates of re-infection outcomes.

Systematic review registration: PROSPERO 2015: CRD42015016664

Keywords: Infection, Hip replacement, Revision, One-stage, Two-stage, Individual participant data, Meta-analysis

\footnotetext{
* Correspondence: setor.kunutsor@bristol.ac.uk

'Musculoskeletal Research Unit, School of Clinical Sciences, Learning and Research Building (Level 1), Southmead Hospital, University of Bristol,

Southmead Road, Bristol BS10 5NB, UK

Full list of author information is available at the end of the article
} 


\section{Background}

Hip replacement is widely used to treat pain associated with diseased or damaged joints. Deep prosthetic infection is an uncommon but serious complication of hip replacement with estimated incidence in the UK population of about $0.70 \%$ [1]. Prosthetic joint infection occurring within 2 years of hip replacement is mainly a consequence of the surgical intervention [2] and are commonly associated with extreme pain, restricted movement, feelings of isolation, insecurity and hopelessness and may even lead to death [3,4]. Treatment options for prosthetic hip infection include the following: surgical removal of dead, damaged and infected tissue (debridement) with prosthesis retention and long-term antibiotic treatment; debridement and the exchange of modular components; one-stage revision; two-stage revision; excision of the joint replacement; or amputation. One-stage surgical revision involves prosthesis removal, debridement, antibiotic treatment and joint replacement in the same surgical operation. In the two-stage revision, a temporary 'spacer' may be fitted, which is replaced in the second operation typically 2 to 6 months later. The two-stage revision is used commonly and has been traditionally regarded as more effective in treating infection [5,6]. In England and Wales, about 500 hip revision procedures per year following prosthetic joint infection are carried out, with 30\% being onestage revision, $64 \%$ being two-stage and $6 \%$ being excision [7]. Despite the opportunities for additional microbial strategies [8] with the two-stage procedure, patients who undergo this procedure require additional hospital admissions, undergo further major surgery and experience considerable pain and disability during the period between operations and sometimes after the revision [9]. A twostage revision may also cost about $70 \%$ more than a onestage revision [10]. There is an increasing interest in the use of the single-stage revision as it may be associated with significantly less morbidity and disability; may eliminate the requirement for prolonged stay in the hospital; and overall healthcare costs of this procedure may be less than the two-stage procedure. There is currently uncertainty regarding the best treatment option.

There have been no randomised controlled trials comparing one- and two-stage revision procedures. However, several observational studies have assessed re-infection outcomes following the one-stage or two-stage surgical revisions and have reported inconsistent results. Wolf and colleagues conducted a literature review in 2008 and used a decision analysis to compare the one- and twostage revision strategies [11]. In their search of only MEDLINE and the American and British editions of The Journal of Bone and Joint Surgery, 11 two-stage (321 patients) and eight one-stage (576 patients) studies were included in the review. In pooled analysis, they reported an increased re-infection rate after one-stage (12.3\%) compared to two-stage revision (6.5\%) of infected total hip replacements [11]. There was however a higher mortality rate associated with the two-stage compared to one-stage. Using a Markov cohort simulation decision analysis, they reported that the overall balance of risk and benefit favours the one-stage approach in the treatment of infection after a total hip replacement. Lange and colleagues in their systematic review and metaanalysis included a total of 36 published studies (comprising 375 one-stage and 929 two-stage patients), identified from a search of PUBMED, EMBASE, Cochrane Library and the WHO International Clinical Trials Registry Platform databases till 2010 [12]. Re-infection rates of $13.1 \%$ and $10.4 \%$ were reported for one- and two-stage revision strategies, respectively. Our group has also recently assessed re-infection outcomes of one- and two-stage revision of infected hip replacements using published studies that included populations representative of patients in routine clinical practice [13]. In a search of EMBASE, MEDLINE and Cochrane databases, 11 one-stage (1,225 patients) and 28 two-stage (1,188 patients) studies were included in the review. Pooled random-effects metaanalysis yielded a lower re-infection rate at 2 years for one-stage-revision (8.6\%) compared to a two-stage revision (10.2\%), although this difference was not significant. The inconsistent evidence does not conclusively support a specific revision strategy for prosthetic hip infection. In addition, there were several features of these aggregate reviews which limit the validity of the findings. Firstly, none of the reviews performed a detailed quality assessment of the included studies. Secondly, the heterogeneous definition of re-infection outcomes by the studies included did not allow reliable comparison of the findings. Though our review attempted to minimise this by assessing only reinfection outcomes within 2 years of revision surgery, this approach was not possible in all studies. Thirdly, there was substantial heterogeneity amongst contributing studies which could not be adequately explored. Finally, majority of these reviews did not conduct any subgroup analysis or assess the possibility of publication bias.

Given these limitations of aggregate published data, the results of the previous reviews may be misleading and potentially promote inappropriate recommendations for a specific strategy for revision of infected hip prostheses. To compare the effectiveness of one-stage and two-stage revision will require robust evidence from a carefully designed randomised clinical trial. With the low incidence of prosthetic hip infection, an appropriate definitive randomised trial with re-infection as the primary outcome is currently unlikely. In the absence of such a clinical trial, we propose the Global Infection Orthopaedic Management (INFORM) collaboration, a worldwide collaborative systematic review and metaanalysis of individual participant data (IPD) to address 
the existing uncertainties in the previous reviews. This will enable a consistent approach to the definition of reinfection outcomes, more detailed analyses under a broader range of circumstances, exploration of potential sources of heterogeneity and produce much more valid and precise estimates of re-infection outcomes.

\section{Aims and objectives}

The main aim of this worldwide collaborative systematic review and meta-analysis of IPD is to compare the effectiveness of one- and two-stage surgical revision to prevent re-infection following prosthetic hip infection. Specific objectives are as follows:

1. To compare characteristics of patients undergoing one-stage or two-stage revision surgery following prosthetic hip infection

2. To assess average time to re-infection following onestage or two-stage revision surgery

3. To assess incidence of re-infection (primary outcome) for one-stage or two-stage revision surgery

4. To compare risk of re-infection for one-stage compared to two-stage revision surgery following adjustment for potential confounders

5. To compare risk of re-infection for one-stage compared to two-stage revision surgery in clinically relevant subgroups

6. To compare other outcome measures such as patient reported function, readmission, pain and death (when data permits).

\section{Methods}

We will conduct this systematic review and IPD metaanalysis using a predefined protocol and in accordance with methods recommended by the IPD Meta-analysis Methods Group of the Cochrane Collaboration [14] and other published guidelines [15]. Results will be reported in accordance with relevant aspects of PRISMA and MOOSE guidelines [16,17]. In accordance with the PRISMA 2015 statement $[18,19]$, in the event of an important protocol amendment - the date of the amendment, description of the amendment and rationale for amendment will be provided. A revised protocol will be generated which will list the specific amendments made to the previous version. The lead investigator will be responsible for approving and supervising the documentation and implementation of the amendments.

\section{Data sources and search strategy}

We will update the literature search to identify new studies published since the completion of our systematic review [13] which compared the effectiveness of onestage and two-stage revision for re-infection outcomes following prosthetic hip infection. We will systematically search for longitudinal studies (retrospective, prospective or randomised controlled trials) reporting re-infection outcomes following one- or two-stage surgical revision of infected hip prosthesis in MEDLINE, EMBASE, Web of Science, Cochrane Database of Systematic Reviews, Cochrane Central Register of Controlled Trials and the WHO International Clinical Trials Registry Platform from March 2011 (date of our last search for the previous review) to date. The computer-based searches will combine free and $\mathrm{MeSH}$ search terms and combination of keywords related to hip replacement, infection and revision with focus on one- and two stage revision surgeries. There will be no restrictions on language. Reference lists of retrieved articles will be manually scanned for all relevant additional studies and review articles. An additional Microsoft Word file shows the search strategy in more detail [see Additional file 1]. To minimise publication bias, information on studies in progress (Clinical Trials.gov) and studies reported in the grey literature [Systems for Information in Grey Literature (SIGLE) and Dissertation abstracts] will be sought. See Figure 1 for preliminary PRISMA flow of studies.

\section{Eligibility criteria}

Studies will be included if they involve unselected patients with prosthetic hip infection (over 18 years of age) treated exclusively by one-stage or two-stage revision and had at least been followed up for 2 years for reinfection outcomes after revision surgery. Studies that report case series of methods in selected group of patients (such as subsamples of patients who received revision in one or two stages or patients with a specific infection), did not include patients with less than 2 years of follow-up, and studies with less than ten participants will be excluded from the review.

\section{Study selection and quality assessment}

Two investigators will independently review titles and abstracts for eligibility. If either reviewer determines that a study may be eligible based on title or abstract review, then a full-text article review will be completed. Each article will be assessed using the inclusion criteria above and any disagreement regarding eligibility of an article will be discussed, and agreement reached by consensus with a third reviewer. All studies in languages other than English will be translated into English. Methodological quality will be assessed based on the Methodological Index for Non-Randomised Studies (MINORS), a validated instrument which is designed for assessment of methodological quality of non-randomised studies in surgery [20]. For non-comparative studies, it uses eight pre-defined domains namely: a clearly stated aim, inclusion of consecutive patients, prospective collection of data, endpoints appropriate to the aim of the study, 


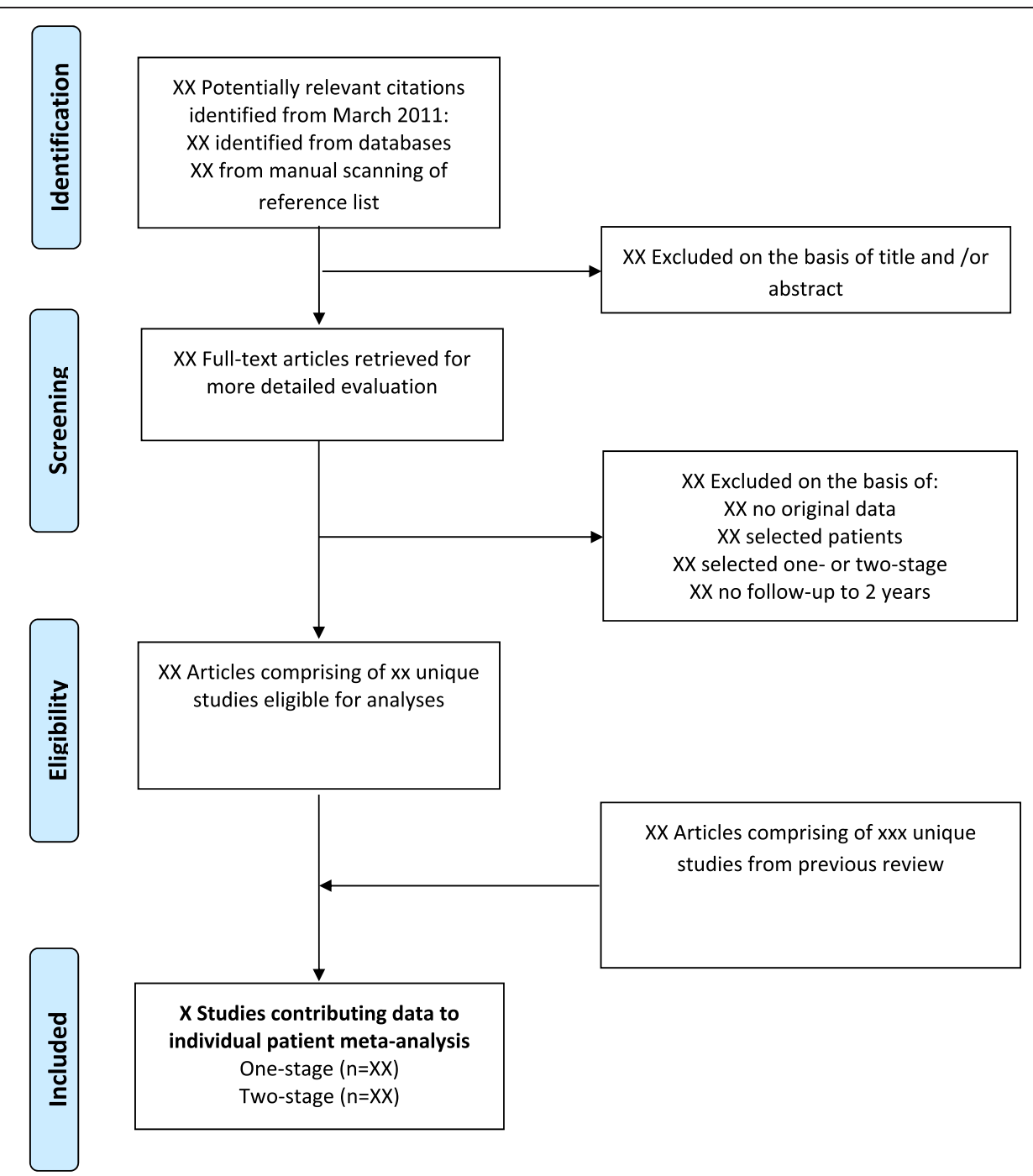

Figure 1 Draft PRISMA flow diagram.

unbiased assessment of the study endpoint, follow-up period appropriate to the aim of the study, loss to follow-up less than $5 \%$ and prospective calculation of the study size. For each item, MINORS assigns 0 for not reported, 1 for reported but inadequate, or 2 for reported and adequate. The global ideal score is 16 .

\section{Establishment of the Global INFORM collaboration}

Investigators of eligible studies will be invited to join the collaboration by providing us with individual level data. We will identify contact information from the published studies or an online search. Each principal investigator will be contacted and provided with the protocol and a cover letter. Based on our recent published review [13], we have identified key studies that may potentially contribute data to this collaboration. We are also in the process of updating our previous review as several important studies have been published since its publication.
A number of authors of studies have expressed initial interest to collaborate in this effort [21-28] (Table 1).

\section{Data collection}

Investigators will be provided with a list of relevant study variables that could be used in the analyses (Table 2), and data dictionaries will be requested. We will accept data in all formats but preferably in STATA, SPSS or Microsoft Excel. Individual level data collected will be cleaned, coded and entered into a single STATA database (Stata Corp, College Station, TX, USA).

\section{Statistical analysis}

Re-infection within 2 years of hip revision surgery will be used as the primary outcome. Secondary outcomes will include further treatments (such as re-revision surgery), pain, functional outcomes and death. No formal sample size requirements are necessary for the meta- 
Table 1 Characteristics of studies with provisional support to share individual level data

\begin{tabular}{|c|c|c|c|c|c|c|c|c|c|c|}
\hline $\begin{array}{l}\text { Lead author, publication date } \\
\text { (reference number) }\end{array}$ & Location & Year of study & $\begin{array}{l}\text { Mean } / \text { median } \\
\text { age (years) }\end{array}$ & $\%$ male & $\begin{array}{l}\text { Follow-up } \\
\text { mean/median } \\
\text { (months) }\end{array}$ & $\begin{array}{l}\text { Type of } \\
\text { re-implantation }\end{array}$ & $\begin{array}{l}\text { Use of } \\
\text { spacer }\end{array}$ & $\begin{array}{l}\text { Number of } \\
\text { re-infections }\end{array}$ & $\begin{array}{l}\text { Number of } \\
\text { participants }\end{array}$ & $\begin{array}{l}\text { Type of } \\
\text { revision }\end{array}$ \\
\hline Elson et al. 1993 [21] & UK & NS & NS & NS & NS & NS & NA & 33 & 235 & One-stage \\
\hline Whittaker et al. 2009 [22] & UK & 1998 to 2003 & 69.0 & 49.0 & 49.0 & Both & Spacer & 6 & 43 & Two-stage \\
\hline Cabrita et al. 2007 [23] & Brazil & 1996 to 2003 & 54.6 & 58.0 & 48.0 & NS & Spacer & 4 & 38 & Two-stage \\
\hline Ritter et al. 2010 [25] & USA & 1969 to 2004 & 66.2 & 52.0 & 82.8 & NS & NS & 5 & 17 & Two-stage \\
\hline De Man et al. 2011 [24] & Switzerland & 1985 to 2004 & 70.0 & 57.0 & 45.6 & NS & NS & 1 & 55 & One-stage \\
\hline Neumann et al. 2012 [26] & Austria & 2000 to 2008 & 25 to $84^{a}$ & 56.8 & 67.0 & Cementless & Spacer & 1 & 44 & Two-stage \\
\hline Schwarzkopf et al. 2014 [27] & USA & 2001 to 2011 & 62.3 & 46.9 & 32.4 & Cemented & Spacer & 3 & 62 & Two-stage \\
\hline Zeller et al. 2014 [28] & France & 2002 to 2010 & 71.0 & 58.0 & 41.6 & Cementless & NA & 6 & 157 & One-stage \\
\hline
\end{tabular}

NA, not applicable; NS, not stated; Both, some participants had cemented re-implantation and others cementless.

${ }^{\mathrm{a} A g e}$ range. 
Table 2 Study variables to be requested for individual participant data meta-analysis

\begin{tabular}{ll}
\hline Broad classification & Variables \\
\hline Socio-demographic characteristics & $\begin{array}{l}\text { Country in which study was carried out, age, sex, body mass index (weight and height) and } \\
\text { smoking status }\end{array}$ \\
Past medical/surgical history & $\begin{array}{l}\text { Previous hip surgery, other joint surgery and comorbidities (for example, history of diabetes), } \\
\text { Charnley classification, Charlson index, ASA grade }\end{array}$ \\
Infection characteristics before revision surgery & $\begin{array}{l}\text { Duration between index implantation and occurrence/diagnosis of infection, duration between } \\
\text { diagnosis of infection and revision surgery }\end{array}$ \\
Baseline laboratory data for infection & $\begin{array}{l}\text { CRP, ESR, leucocytes, causative organism, neutrophil count, white cell count, synovial fluid white } \\
\text { cell count, IL-6 }\end{array}$ \\
Characteristics of surgical revision & $\begin{array}{l}\text { Date of revision, type of re-implantation, type of fixation (cemented or uncemented), use of spacer, } \\
\text { type of spacer (static or articulating), use of antibiotics in cement or spacer, time interval between } \\
\text { stages for two-stage procedure, diagnosis of re-infection, date of diagnosis of re-infection or date of } \\
\text { last follow-up for participants without re-infection, time to re-infection or last follow-up (days or } \\
\text { years), antibiotics used and duration of antibiotics } \\
\text { One- or two-stage revision }\end{array}$ \\
\hline Intervention
\end{tabular}

ASA, American Society of Anaesthesiologists; CRP, C-reactive protein; ESR, erythrocyte sedimentation rate; IL-6, interleukin- 6 .

analysis. Primary analyses will be conducted comparing the one-stage to the two-stage (reference category) procedure. IPD analyses will be based on Cox proportional hazard $(\mathrm{PH})$ models estimated for each study separately. All PH models will be stratified by sex and adjusted for age, body mass index, comorbidities and other potential confounders. Study-specific log hazard ratios (HRs) will be combined using random-effects meta-analysis. Betweenstudy heterogeneity will be quantified by the $\mathrm{I}^{2}$ statistic. Supplementary analyses will combine log HRs using fixedeffects meta-analysis. Given the distortions (for example, non-convergence of statistical models) that can appear in analyses involving small strata (such as studies contributing less than ten outcomes to a specific analysis) when using two-stage $\mathrm{PH}$ models, a single-stage $\mathrm{PH}$ model which maintains clustering of the data will also be considered. In subsidiary analyses, a propensity score approach will also be used to compare re-infection outcomes between the two types of revisions. Comparison of the oneto two-stage approach for re-infection incidence may vary under different circumstances. There is therefore no reason to expect a priori that the results will be similar for example in patients with/without a history of diabetes or in regions that have different underlying infection patterns. It is therefore important to investigate the comparisons according to different level characteristics. Therefore, primary analyses will be repeated to calculate HRs for reinfection according to different individual and study level characteristics such as sex, age groups, body mass index, comorbidities, major geographical regions (that is, Europe/ North America/Australia/NZ, South Asia and East Asia) and study quality (low versus high quality studies). Sensitivity analysis will include determining HRs for reinfection on exclusion of low-quality studies. The potential impact of publication bias and unavailable data will be explored in accordance with methods proposed by Ahmed and colleagues [29]. Funnel plots will also be constructed to examine the likelihood of publication bias. All analyses will be conducted using Stata version 13 (Stata Corp, College Station, TX, USA).

\section{Ethical issues}

We will not seek ethical approval for this systematic and IPD meta-analysis, because we will be collecting and synthesising the same data from previously published studies in which informed consent and ethical approval has already been obtained by the study investigators (which will be confirmed in the published study and in writing). Secondly, our systematic review and IPD metaanalysis will be addressing similar questions to the research question for which the data were collected. Thirdly, we will request investigators to submit anonymised datasets. Finally, our Research Ethics Committee has confirmed that ethical approval is not required for the present study, given the above reasons. Our research is supported by an established patient forum [30], and we will seek advice on issues relating to review conduct and dissemination of results.

\section{Proposed collaborative publication arrangements}

It is proposed that the following collaborative arrangements should be used in this effort: 1) scientific papers will be published in the name of a collaborative group (for example, 'Global Infection Orthopaedic Management Collaboration'), 2) the members of a writing committee are to be listed at the end of the paper with a small number of representatives (for example, three from each of the contributing studies sharing data), 3) the sequence of names in the writing committee is to be arranged to help demonstrate the collaborative nature of this effort (for example, each study will be able to include one 'starred' joint first author and one 'starred' joint final author), 4) in addition to the researchers listed in the writing committee and the analytical subcommittee, 
it may be possible to list under a separate heading additional scientists who have made contributions to this effort (for example, under the heading 'Investigators', it may be possible to list principal investigators from each of the component studies, subject to the approval of the paper's target journal, which has not yet been determined). The Cochrane IPD meta-analysis method group recommends a collaborators' meeting for preliminary presentation of meta-analyses [31]. We anticipate that this will take the form of a teleconference but will explore the feasibility of holding a workshop locally or at an appropriate international conference.

\section{Discussion}

The present analysis will differ from the previous reviews in the following main ways: (i) access to individual level data should enable a consistent approach to the definition of the primary outcome (2-year incidence of re-infection), a common approach across studies to statistical analyses and a consistent approach to adjustment for potential confounders, a greater ability to explore and identify sources of between-study heterogeneity, comparison of the one- to two-stage revision for re-infection incidence under different level characteristics and ability to assess secondary outcomes such as patient-reported function, pain and death; (ii) statistical power - as the present analysis will involve several-fold more participants, it should be substantially more powerful and precise than previous individual studies and reviews; (iii) generalisability - as it is anticipated to include studies with populations representative of patients in routine clinical practice, the results from the present meta-analysis should be more generalisable. Moreover, inclusion in the proposed analysis of key prospective studies worldwide should help avoid biases due to selective inclusion of studies and enhance the generalisability of the study results.

\section{Registration}

The protocol for the systematic review and IPD metaanalysis has been registered with PROSPERO, the international prospective register of systematic reviews, (PROSPERO 2015: CRD42015016664). Available from http://www.crd.york.ac.uk/PROSPERO/display_record.asp? ID=CRD42015016664.

\section{Additional file}

Additional file 1: Search strategy for MEDLINE.

\section{Abbreviations}

HR: hazard ratio; INFORM: Infection Orthopaedic Management; IPD: individual participant data; MINORS: Methodological Index for Non-Randomised Studies; PH: proportional hazards; PRISMA: Preferred Reporting Items for Systematic Reviews and Meta-Analyses.

\section{Competing interests}

The authors declare they have no competing interests.

\section{Authors' contributions}

SKK, ADB and AWB contributed to the conception and design of the systematic review and meta-analysis. SKK, MRW, JW, AT, IS, AT, SJ, MW, BB, TB, J-PW, AWB and ADB will be involved in the acquisition of data. SKK and ADB drafted this protocol. All authors provided critical revisions of the protocol and approved submission of the final manuscript.

\section{Funding}

This article presents independent research funded by the National Institute for Health Research (NIHR) under its Programme Grants for Applied Research programme (RP-PG-1210-12005). The views expressed in this article are those of the authors and not necessarily those of the NHS, the NIHR or the Department of Health.

\section{Author details}

${ }^{1}$ Musculoskeletal Research Unit, School of Clinical Sciences, Learning and Research Building (Level 1), Southmead Hospital, University of Bristol, Southmead Road, Bristol BS10 5NB, UK. ${ }^{2}$ North Bristol NHS Trust, Bristol BS10 5NB, UK. ${ }^{3}$ Royal Devon and Exeter NHS Foundation Trust, Exeter EX2 7JU, UK. ${ }^{4}$ Northern General Hospital, Sheffield Teaching Hospitals NHS Trust, Sheffield S5 7AU, UK. ${ }^{5}$ Oxford University Hospitals NHS Trust, Oxford OX3 9DU, UK. ${ }^{6}$ Cardiff and Vale University Health Board, Llandough CF5 2LD, UK. ${ }^{7}$ The Robert Jones and Agnes Hunt Orthopaedic Hospital NHS Foundation Trust, Oswestry SY10 7AG, UK. ${ }^{8}$ Wrightington, Wigan and Leigh NHS Foundation Trust, Wigan WN6 9EP, UK.

Received: 2 March 2015 Accepted: 9 April 2015

Published online: 25 April 2015

\section{References}

1. Public Health England. Surveillance of surgical site infections in NHS hospitals in England, 2013/14. London: Public Health England, December 2014. Available from: www.gov.uk/phe.

2. Zimmerli W, Trampuz A, Ochsner PE. Prosthetic-joint infections. N Engl J Med. 2004;351(16):1645-54. doi:10.1056/NEJMra040181.

3. Andersson AE, Bergh I, Karlsson J, Nilsson K. Patients' experiences of acquiring a deep surgical site infection: an interview study. Am J Infect Control. 2010;38(9):711-7.

4. Hunter G, Dandy D. The natural history of the patient with an infected total hip replacement. J Bone Joint Surg Am. 1977;59-B(3):293-7.

5. Cooper HJ, Della Valle CJ. The two-stage standard in revision total hip replacement. Bone Joint J. 2013;95-B(11 Suppl A):84-7. doi:http://dx.doi.org/ 10.1302/0301-620X.95B11.32906.

6. Bottner F, Sculco TP. Infection in revision total hip arthroplasty. Tech Orthop. 2001;16(3):310-22.

7. National Joint Registry for England and Wales: 10th Annual Report. Hemel Hempstead: NJR Centre. 2013.

8. Hsieh P-H, Shih C-H, Chang Y-H, Lee MS, Shih H-N, Yang W-E. Two-stage revision hip arthroplasty for infection: comparison between the interim use of antibiotic-loaded cement beads and a spacer prosthesis. J Bone Joint Surg Am. 2004;86-A(9):1989-97.

9. Cahill JL, Shadbolt B, Scarvell JM, Smith PN. Quality of life after infection in total joint replacement. J Orthop Surg. 2008;16(1):58-65.

10. Klouche S, Sariali E, Mamoudy P. Total hip arthroplasty revision due to infection: a cost analysis approach. Orthop Traumatol Surg Res. 2010;96(2):124-32. doi:10.1016/j.otsr.2009.11.004.

11. Wolf CF, Gu NY, Doctor JN, Manner PA, Leopold SS. Comparison of one and two-stage revision of total hip arthroplasty complicated by infection: a Markov expected-utility decision analysis. J Bone Joint Surg Am. 2011;93-A(7):631-9

12. Lange J, Troelsen A, Thomsen RW, Soballe K. Chronic infections in hip arthroplasties: comparing risk of reinfection following one-stage and twostage revision: a systematic review and meta-analysis. Clin Epidemiol. 2012;4:57-73. doi:10.2147/CLEP.S29025

13. Beswick A, Elvers K, Smith A, Gooberman-Hill R, Lovering A, Blom A. What is the evidence base to guide surgical treatment of infected hip prostheses? Systematic review of longitudinal studies in unselected patients. BMC Med. 2012;10(1):18 
14. Cochrane Collaboration Individual Patient Data (IPD) Meta-analysis Methods Group. 2007. http://ipdmamg.cochrane.org/resources.

15. Riley RD, Lambert $P C$, Abo-Zaid G. Meta-analysis of individual participant data: rationale, conduct, and reporting. BMJ. 2010;340:521-5. doi:10.1136/ bmj.c221.

16. Stroup DF, Berlin JA, Morton SC, Olkin I, Williamson GD, Rennie D, et al. Meta-analysis of observational studies in epidemiology. JAMA. 2000;283(15):2008-12. doi:10.1001/jama.283.15.2008.

17. Moher D, Liberati A, Tetzlaff J, Altman DG. Preferred reporting items for systematic reviews and meta-analyses: the PRISMA statement. PLoS Med. 2009;6(7), e1000097. doi:10.1371/journal.pmed.1000097.

18. Moher D, Shamseer L, Clarke M, Ghersi D, Liberati A, Petticrew M, et al. Preferred reporting items for systematic review and meta-analysis protocols (PRISMA-P) 2015 statement. Syst Rev. 2015;4:1. doi:10.1186/2046-4053-4-1.

19. Shamseer L, Moher D, Clarke M, Ghersi D, Liberati A, Petticrew M, et al. Preferred reporting items for systematic review and meta-analysis protocols (PRISMA-P) 2015: elaboration and explanation. BMJ. 2015;349:g7647. doi:10.1136/bmj.g7647.

20. Slim K, Nini E, Forestier D, Kwiatkowski F, Panis Y, Chipponi J. Methodological index for non-randomized studies (MINORS): development and validation of a new instrument. ANZ J Surg. 2003;73(9):712-6.

21. Elson RA. Exchange arthroplasty for infection. Perspectives from the United Kingdom. Orthop Clin North Am. 1993;24(4):761-7.

22. Whittaker JP, Warren RE, Jones RS, Gregson PA. Is prolonged systemic antibiotic treatment essential in two-stage revision hip replacement for chronic gram-positive infection (Journal of Bone and Joint Surgery - Series B (2009) 91-B, (44-51). J Bone Joint Surg (Br). 2009;91(5):700.

23. Cabrita HB, Croci AT, Camargo OP, Lima AL. Prospective study of the treatment of infected hip arthroplasties with or without the use of an antibiotic-loaded cement spacer. Clinics. 2007;62(2):99-108.

24. De Man FH, Sendi P, Zimmerli W, Maurer TB, Ochsner PE, Ilchmann T. Infectiological, functional, and radiographic outcome after revision for prosthetic hip infection according to a strict algorithm. Acta Orthop. 2011;82(1):27-34.

25. Ritter MA, Farris A. Outcome of infected total joint replacement Orthopedics. 2010;33(3). doi:10.3928/01477447-20100129-09.

26. Neumann DR, Hofstaedter T, List C, Dorn U. Two-stage cementless revision of late total hip arthroplasty infection using a premanufactured spacer Journal of Arthroplasty. 2012;27(7):1397-401. doi:http://dx.doi.org/10.1016/j. arth.2011.10.022.

27. Schwarzkopf R, Mikhael B, Wright E, Estok 2nd DM, Katz JN. Treatment failure among infected periprosthetic total hip arthroplasty patients. Open Orthop J. 2014:8:118-24. doi:10.2174/1874325020140515002.

28. Zeller V, Lhotellier L, Marmor S, Leclerc P, Krain A, Graff W, et al. One-stage exchange arthroplasty for chronic periprosthetic hip infection: results of a large prospective cohort study. J Bone Joint Surg - Am Vol. 2014;96(1):e1. doi:http://dx.doi.org/10.2106/JBJS.L.01451.

29. Ahmed I, Sutton AJ, Riley RD. Assessment of publication bias, selection bias, and unavailable data in meta-analyses using individual participant data: a database survey. BMJ. 2012;344. doi:10.1136/bmj.d7762.

30. Gooberman-Hill R, Burston A, Clark E, Johnson E, Nolan S, Wells V, et al. Involving patients in research: considering good practice. Musculoskeletal care. 2013;11(4):187-90.

31. Stewart LA, Tierney JF, Clarke M. Chapter 19: reviews of individual patient data. In: Higgins JPT, Green S, editors. Cochrane Handbook for Systematic Reviews of Interventions Version 5.1.0 (updated March 2011): The Cochrane Collaboration. 2011.

\section{Submit your next manuscript to BioMed Central and take full advantage of:}

- Convenient online submission

- Thorough peer review

- No space constraints or color figure charges

- Immediate publication on acceptance

- Inclusion in PubMed, CAS, Scopus and Google Scholar

- Research which is freely available for redistribution 